\title{
Design and Modal Analysis of Photonic Crystal Fiber for Dispersion Compensation over Broadband Range
}

\author{
Madhavi Waghmare ${ }^{1}$, K.T.V.Reddy ${ }^{2}$, \\ ${ }^{1 .}$ Research Scholar, Department of Electronics and Telecommunication Engineering, Sardar Patel Institute of \\ Technology, University of Mumbai, Mumbai, India. ${ }^{2}$ Pranveer Singh Institute of Technology, Kanpur, India \\ (01madhavi@gmail.com; ktvr@psit.ac.in)
}

\begin{abstract}
In this paper we have presented the four new investigations using different structures of Photonic Crystal Fiber (PCF) for broadband communication applications and narrows down on the design which can provide largest flat negative dispersion. The new structure model is optimized based on the combination of modal properties and dispersion compensation. The results were recorded over a transmission frequency range from 190.95 THz to $245.73 \mathrm{THz}$ i.e. $1.22 \mu \mathrm{m}$ to $1.57 \mu \mathrm{m}$ with a dispersion $-704.62 \mathrm{ps} / \mathrm{nm} / \mathrm{km}$. The results obtained from the new different PCF structures are compared with reported results for different 2D PCF models so far.
\end{abstract}

Index Terms - Dispersion Compensation, Photonic Crystal Fiber, Refractive Index, Confinement loss.

\section{INTRODUCTION}

Dispersion is a key factor for limiting the development in rapid transmission rate in communication field using optic fiber. For high speed and large bandwidth application in optic fiber communication, dispersion characteristic should be uniform or have minimum slope (flattened) throughout the wavelength [1]. In the literature, design of different 2D PCF structures by compensating dispersion by changing geometric parameters of the structure and Refractive Index (RI) have been reported. However, conventional hexagonal PCF does not have air hole at the center of the core. In hexagonal PCF structures may have single air hole or array of air holes at the core.

Feroza Begum et. al. [2] studied conventional hexagonal PCF structure for negative dispersion where $\mathrm{D}=-100 \mathrm{ps} / \mathrm{nm} / \mathrm{km}$ at $1550 \mathrm{~nm}$ wavelength with high losses. Makoui et. al. [3] used generic algorithm to minimize pulse broadening over large wavelength. Varshney et.al. [4] used microstructure of PCF for residual dispersion compensation to achieve flattened negative dispersion. They reported ultra-flattened negative dispersion of $-98.3 \mathrm{ps} / \mathrm{nm} / \mathrm{km}$ and $\Delta \mathrm{D}=1.1 \mathrm{ps} / \mathrm{nm} / \mathrm{km}$ over $\mathrm{S}$ to L wavelength band i.e. wavelength bandwidth of $1.48 \mu \mathrm{m}$ to $1.63 \mu \mathrm{m}$. Similarly, Franco et. al. [5] used microstructure of PCF and investigated ultra-flattened negative dispersion of $-179 \mathrm{ps} / \mathrm{nm} / \mathrm{km}$ and $\Delta \mathrm{D}=2.1 \mathrm{ps} / \mathrm{nm} / \mathrm{km}$ over $\mathrm{S}$ to $\mathrm{U}$ wavelength band i.e. wavelength bandwidth of 1.48 to $1.675 \mu \mathrm{m}$. The design by Silva et.al. [6] demonstrates higher average dispersion $-212 \mathrm{ps} / \mathrm{nm} / \mathrm{km}$ and $\Delta \mathrm{D}=11.9$ $\mathrm{ps} / \mathrm{nm} / \mathrm{km}$ over $\mathrm{E}$ to $\mathrm{U}$ wavelength band i.e. wavelength bandwidth of $1.35 \mu \mathrm{m}$ to $1.7 \mu \mathrm{m}$ and the 
design has been studied with Ge doped core by using genetic algorithm. Asiful Islam et. al. [7] used equiangular spiral PCF structure and achieved absolute dispersion variation of $-227 \mathrm{ps} / \mathrm{nm}-\mathrm{km}$ with $\Delta \mathrm{D} \sim 11 \mathrm{ps} / \mathrm{nm}-\mathrm{km}$ over frequency range $178.98 \mathrm{THz}$ to $202.56 \mathrm{THz}$. Tee et. al. [8] used PCF in PCF structure and achieved residual dispersion compensation $-457.4 \mathrm{ps} / \mathrm{nm} / \mathrm{km}$ over the range larger than E to $\mathrm{U}$ band i.e. (177.3 THz to $220 \mathrm{THz}$ ) and $\Delta \mathrm{D} \sim 11.9 \mathrm{ps} / \mathrm{nm} / \mathrm{km}$. All these studies were carried out on a 2D PCF with uniform air hole structures for high speed and broadband transmission. However, there is still a possibility to achieve more negative dispersion and/or near zero confinement loss over broadband optical frequency range. Higher flattened negative dispersion indicates good dispersion compensation and almost zero confinement loss resulting in enhancement of fiber bandwidth.

In this paper we explained the investigations of different new PCF structures which can provide very large negative dispersion. Four new PCF structures are designed to compensate dispersion of propagation of light with nearly zero confinement loss. In these structures, inner circular air hole array with different geometry is used to reduce dispersion. In this paper we implemented and studied the effect of the defect introduction in the newly designed PCF structures within transmission spectrum range from $190.95 \mathrm{THz}$ to $245.73 \mathrm{THz}$ i.e. $1.22 \mu \mathrm{m}$ to $1.57 \mu \mathrm{m}$ with $-704.62(\mathrm{ps} / \mathrm{nm} / \mathrm{km})$ average large negative dispersion.

\section{MODELLING OF A PHOTONIC CRYSTAL FIBER STRUCTURE}

PCF can have air hole structure of any shape. Commonly two different forms of lattice are used. One with square lattice and other with triangular lattice. PCF has gathered more attention because of their special characteristics in triangular lattice of air holes [9]. Research reports have studied conventional hexagonal PCF and circular PCF structures so far. After studying their investigations on these structures, we have tried to model four different new PCF structures where two PCF structures are combined as a new model in the investigation. We have considered hexagonal and circular structures with triangular lattice for investigation purpose. With the help of combination of hexagonal and circular structures for inner and outer region, there are four possibilities of symmetric structures that demonstrate optimal dispersion and confinement loss. Because of two different structures i.e. inner (core region) and outer (Cladding region), there is a possibility to adjust effective refractive index of the structures by adjusting geometric parameters of inner and outer regions. The large effective refractive index difference between core and cladding region strongly affects the chromatic dispersion. The symmetry of structures prevents the existence of a linear electro-optic effect.

Fig. 1 shows the cross section of the four different structures along with their geometric parameters for better understanding. For outer structure $\mathbf{N}$ denotes the number of air-hole rings and $\mathbf{r}$ is the radius air-hole. If outer structure is hexagon, then a represents pitch and if outer structure is circular, then $\mathbf{d}$ represents the radial distance of the circular structure. For inner structure $\mathbf{N} 1$ represents number of airhole rings and $\mathbf{r} \mathbf{1}$ is the radius of air-hole. If inner structure is hexagon, then $\mathbf{a} 1$ represents pitch and if inner structure is circular, then $\mathbf{d 1}$ represents the radial distance between circular rings. The size of the 
inner structure is considered in such a way that it will fall in the range of fabrication dimension for practical use [10]. The inner structure is core region which helps to produce better negative dispersion. The number of air-hole rings in core region is selected such that it gives large negative dispersion, at the same time the number of air-hole rings at the outer structure is chosen to give low confinement loss [5]. In all the four proposed structures, there are five air-hole rings at the outer structure to reduce the confinement loss.
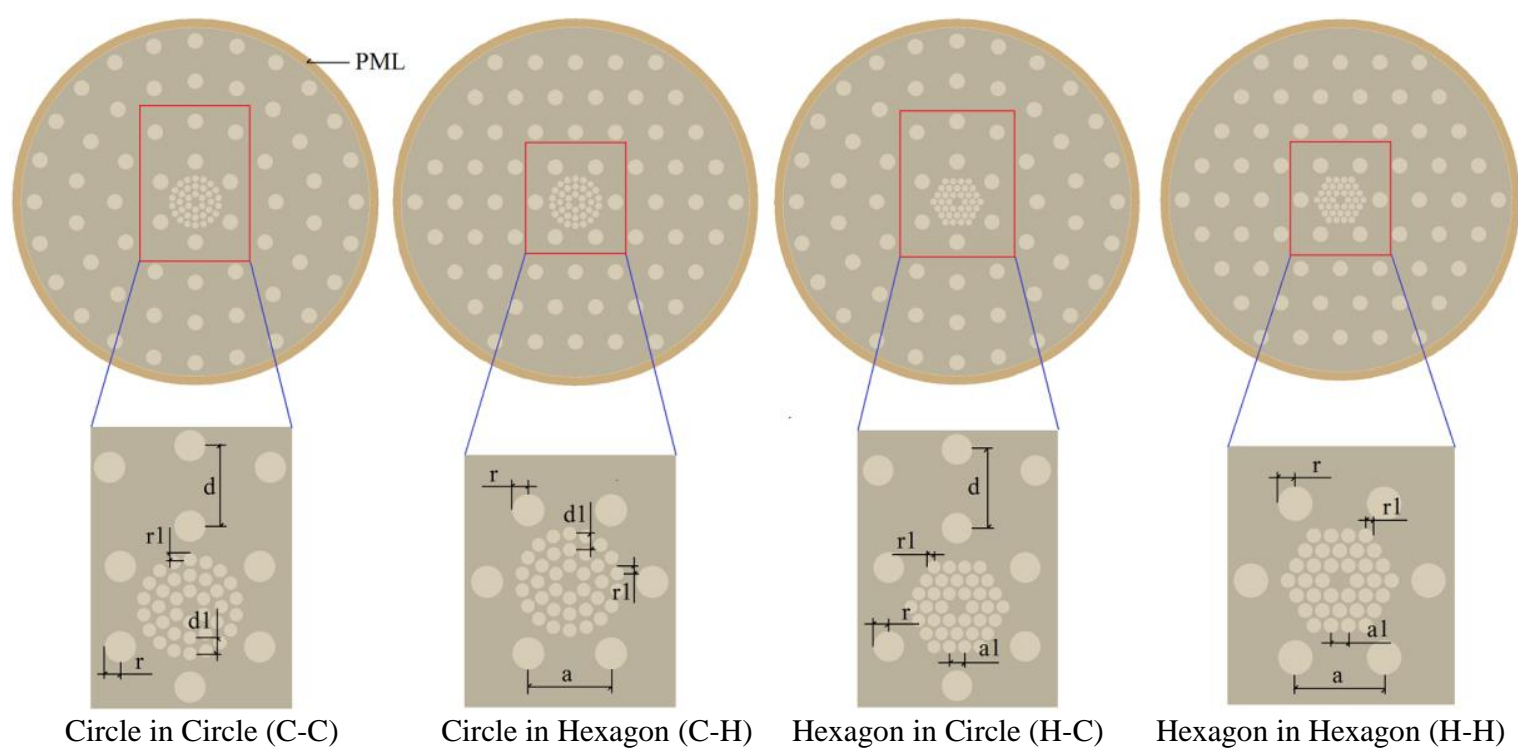

Fig. 1. Cross section of 4 different PCF structure models with geometric parameters

Optimized geometric parameters of these structures are given in table I. These geometric parameters are optimized after rigorous simulation on different combination of parameters of outer and inner structures.

TABLE I. OPTIMIZED GEOMETRIC PARAMETERS OF 4 STRUCTURES

\begin{tabular}{|c|c|c|}
\hline Structure & Outer region & Inner region \\
\hline $\begin{array}{l}\text { Circular in Hexagonal } \\
(\mathrm{C}-\mathrm{H})\end{array}$ & $\begin{array}{l}\text { Hexagon: No. of air-hole rings } \mathrm{N}=5 \\
\mathrm{a}=1.8 \mu \mathrm{m}, \mathrm{r}=0.7 \mu \mathrm{m}\end{array}$ & $\begin{array}{l}\text { Circle: No. of air-hole rings N1 } \\
=5 \\
\mathrm{~d} 1=0.21 \mu \mathrm{m}, \mathrm{r} 1=0.1 \mu \mathrm{m}\end{array}$ \\
\hline $\begin{array}{l}\text { Hexagonal in Hexagonal } \\
\text { (H-H) }\end{array}$ & $\begin{array}{l}\text { Hexagon: No. of air-hole rings } \mathrm{N}=5 \\
\mathrm{a}=1.8 \mu \mathrm{m}, \mathrm{r}=0.7 \mu \mathrm{m}\end{array}$ & $\begin{array}{l}\text { Hexagon: No. of air-hole rings } \\
\mathrm{N} 1=5 \\
\mathrm{a} 1=0.132 \mu \mathrm{m}, \mathrm{r} 1=0.061 \mu \mathrm{m}\end{array}$ \\
\hline $\begin{array}{l}\text { Hexagonal in Circular } \\
\text { (H-C) }\end{array}$ & $\begin{array}{l}\text { Circle: No. of air-hole rings } \mathrm{N}=5 \\
\mathrm{~d}=1.32 \mu \mathrm{m}, \mathrm{r}=0.56 \mu \mathrm{m}\end{array}$ & $\begin{array}{l}\text { Hexagon: No. of air-hole rings } \\
\text { N1 }=5 \\
\text { a1 }=0.132 \mu \mathrm{m}, \mathrm{r} 1=0.061 \mu \mathrm{m}\end{array}$ \\
\hline $\begin{array}{l}\text { Circular in Circular } \\
\text { (C-C) }\end{array}$ & $\begin{array}{l}\text { Circle: No. of air-hole rings } \mathrm{N}=5 \\
\mathrm{~d}=1.32 \mu \mathrm{m}, \mathrm{r}=0.56 \mu \mathrm{m}\end{array}$ & $\begin{array}{l}\text { Circle: No. of air-hole rings } \\
\mathrm{N} 1=5 \\
\mathrm{~d} 1=0.21 \mu \mathrm{m}, \mathrm{r} 1=0.1 \mu \mathrm{m}\end{array}$ \\
\hline
\end{tabular}


The wavelength dependent refractive index of the silica was included in the simulation from Sell Meier equation. Chromatic dispersion is the phenomenon in which the phase velocity of light wave depends on its frequency. Dispersion D in $\mathrm{ps} / \mathrm{nm} / \mathrm{km}$ of a PCF is evaluated by calculating $\eta_{\text {eff }}$ value vs. the wavelength using the eq. 1

$$
D=-\frac{\lambda}{c} \frac{d^{2} R e\left[\eta_{e f f}\right]}{d \lambda^{2}}
$$

where $\mathrm{c}$ is the velocity of light in vacuum, $\lambda$ is wavelength and $\operatorname{Re}\left[\eta_{e f f}\right]$ is the real part of the refractive index. Chromatic dispersion D is also dependent on the geometric parameters like shape of air holes, pitch, and inner circular holes diameter. By optimizing these parameters, suitable guiding properties can be obtained.

Confinement loss $L_{c}$ is the light confinement ability within the core region and is evaluated using eq. 2. The increase of number of air hole rings support the confinement of light in the core region, which results in minimum losses than those with less air hole rings. Also, increasing the air holes radius results in the increasing of the air filling fraction and accordingly decreasing the loss.

$$
L_{c}=\frac{\left(20 \times 10^{6}\right)}{\ln (10)} K_{0} \operatorname{Im}\left[\eta_{\text {eff }}\right]
$$

where $K_{0}=2 \pi / \lambda$ is wave number in free space with the unit $\mathrm{dB} / \mathrm{m}, \operatorname{Im}\left[\eta_{\text {eff }}\right]$ is the imaginary part of the refractive index. From eq. 1 and 2, it is observed that the dispersion is dependent on real value of refractive index and confinement loss is dependent on imaginary part of refractive index with wavelength. Eq. 3 below shows the relation between refractive index and group velocity.

$$
v_{g}=\left(\frac{\partial \mathcal{K}}{\partial \omega}\right)^{-1}=\mathrm{c}\left(\frac{\partial}{\partial \omega}\left(\omega \eta_{e f f}\right)^{-1}=\frac{c}{\eta_{e f f}+\omega \frac{\partial \eta}{\partial \omega}}=\frac{c}{\eta_{g}(\omega)}\right.
$$

where $\eta_{e f f}$ is the refractive index and $\eta_{g}$ is the group index. The wave number $\mathrm{k}$ can be considered as the change in spectral phase per unit length [10].

The Effective modal area Aeff was calculated using equation 4

$$
A_{e f f}=\frac{\left(\iint\left|E^{2}\right| d x d y\right)^{2}}{\iint|E|^{4} d x d y}
$$

The effective core radius is $0.4 \times a$. In particular, the effective nonlinearity of the fiber can be calculated in the core of the fiber. The effective nonlinear coefficient is calculated by equation 5 .

$$
\gamma=\frac{2 \pi \eta_{2}}{\lambda A_{e f f}}
$$

where $\eta_{2}$ is material nonlinear coefficient. In this paper $\eta_{2}$ used as silica fiber value is $2.6 \times 10^{-20} \mathrm{~m}^{2}$ $\mathrm{W}^{-1}$ which determined by fiber designs.

A Finite Difference Time Domain (FDTD) Lumerical solution simulation tool was used to evaluate the performance of these structures. The simulation region was trimmed by applying a perfectly matched layer surrounding the structure. 


\section{RESULTS AND ANALYSIS}

It has been an attempt by every research scholar in the field of fiber optics communication, to produce an optimized PCF structure which enables better negative dispersion and near zero confinement loss. In the proposed four different structures, changes in various combinations of PCF parameters were carried out during simulation till the optimized structure is found. The objective was to achieve better results than the results produced by other research scholars so far.

From fabrication perspective $\pm 2 \%$ manufacturing tolerance is generally considered [11]. Thus each of the structure's geometric parameters were varied up to $\pm 2 \%$ and simulation was carried out. Finally, all structures (without considering any manufacturing inaccuracies) were compared with each other for better dispersion and nearly zero confinement loss.

Fig. 2, 3, 4 and 5 shows the changes in the dispersion values against the selected wavelength range for variation in geometric parameters by $\pm 2 \%$ for all PCF structure models Circle in Circle (C-C), Hexagon in Hexagon $(\mathrm{H}-\mathrm{H})$, Circle in Hexagon $(\mathrm{C}-\mathrm{H})$ and Hexagon in Circle $(\mathrm{H}-\mathrm{C})$ presented. However, one should note that after fabrication, geometric parameters of the cross section does not vary in only one direction i.e. either $+2 \%$ or $-2 \%$. They are normally mixed and therefore the final effect is more or less balanced. Therefore, more weightage should be given to the results carried out for the nominal dimensions of the geometric parameters. Fig. 2, 3, 4 and 5 shows dispersion values for optimized parameters and changing one of the parameters while keeping other parameters constant. Structure $\mathrm{C}-\mathrm{H}$ shows higher negative dispersion range values as compared to other structures considering $\pm 2 \%$ variation in the geometrical parameters.

In fig. 2, C-H structure shows the effect of the $\pm 2 \%$ variation in the parameter of $\mathbf{r} \mathbf{1}$ and $\mathbf{d} \mathbf{1}$ of inner region and $\mathbf{r}$ and $\mathbf{a}$ of outer region on dispersion. For this study, changes were done to only one of the parameters keeping remaining parameters at optimum (nominal) value. For example, variation of \pm $2 \%$ in optimum value of pitch which is $1.8 \mu \mathrm{m}$ considered while keeping other nominal dimensions constant to $\mathrm{r}=0.7 \mu \mathrm{m}, \mathrm{d} 1=0.21 \mu \mathrm{m}$ and $\mathrm{r} 1=0.1 \mu \mathrm{m}$. Similar study was carried out for other three structures also. $\mathrm{A} \pm 2 \%$ variation in $\mathbf{r}$ results in $\pm 3 \%$ change in average dispersion, while $\Delta \mathrm{D}$ increases more than $100 \%$, whereas $\pm 2 \%$ variation in a results the average dispersion by $\sim \pm 2 \%$ with around $50 \%$ increases in $\Delta \mathrm{D}$. Keeping outer parameter at optimum value and varying inner parameter by $\pm 2 \%$ in $\mathbf{r} 1$ results in $\pm 4 \%$ changes in average dispersion with $\sim 60 \%$ increase in $\Delta \mathrm{D}$. And $\pm 2 \%$ variation in $\mathbf{d 1}$ results in $\pm 3 \%$ changes in average dispersion with $\sim 80 \%$ increase in $\Delta \mathrm{D}$.

The variation in these four parameters (one at a time) do not change the shape of the dispersion curve significantly. There is small variation in guided mode profile corresponding to the changes in one of the parameters at any given instance. Similar observation was registered for other three structures also. 


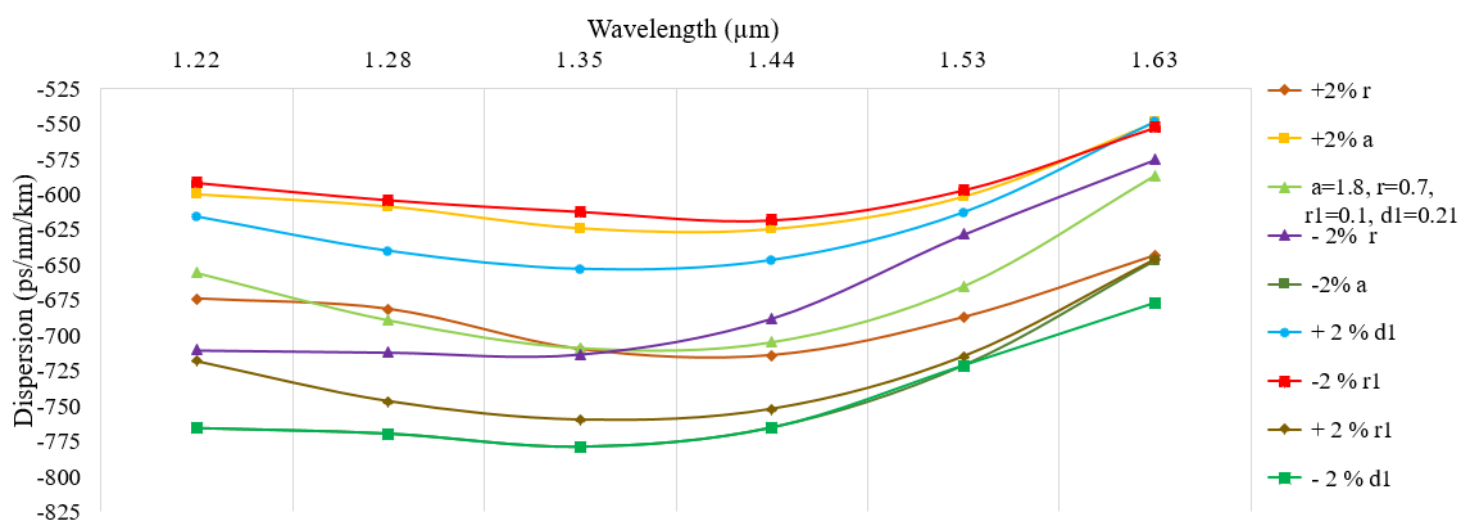

Fig. 2. C-H: Dispersion vs Wavelength

In fig. 3, H-C structure demonstrates sensitivity of dispersion for $\pm 2 \%$ variation in the parameter of $\mathbf{r 1}, \mathbf{a} 1$ of inner region and $\mathbf{r}, \mathbf{d}$ of outer region. From the curve it is seen that $\pm 2 \%$ variation in $\mathbf{r} 1$ results in $\sim \pm 3 \%$ changes in average dispersion with $60 \%$ increase in $\Delta \mathrm{D}$. Similarly, $\pm 2 \%$ variation in a1 results in $\sim \pm 3 \%$ changes in average dispersion with $60 \%$ increase in $\Delta \mathrm{D}$ and $\pm 2 \%$ variation in outer region $\mathbf{r}, \mathbf{d}$ parameters affects $\pm 4 \%$ and $\pm 3 \%$ variation in average dispersion respectively with $80 \%$ and $60 \%$ increase in $\Delta \mathrm{D}$ respectively

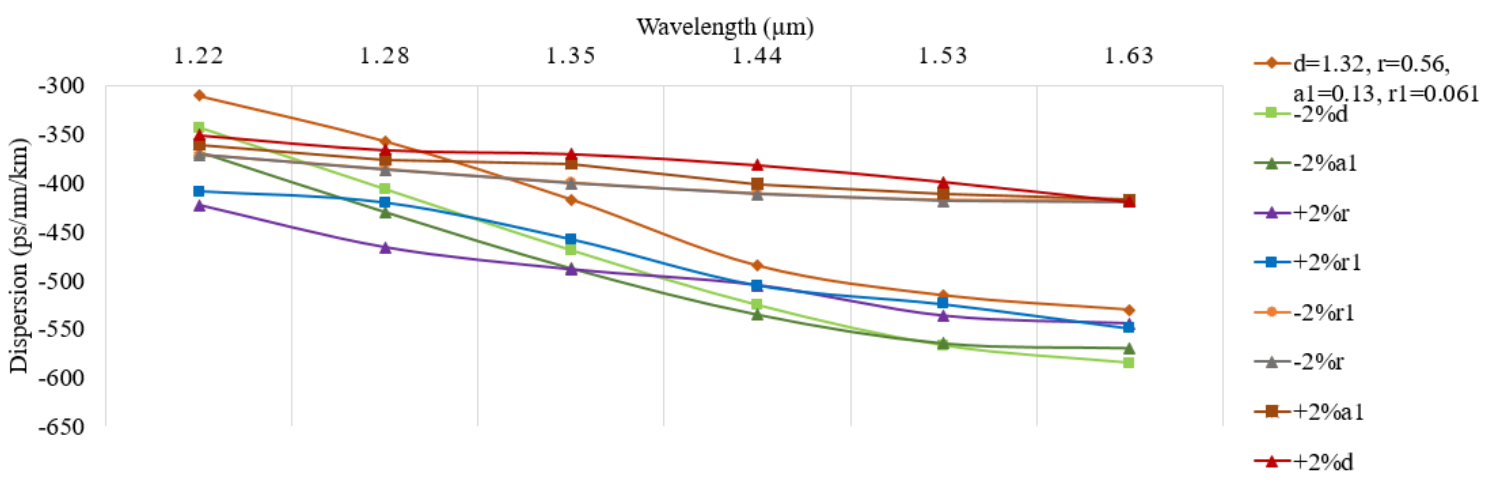

Fig. 3. H-C: Dispersion vs Wavelength

In fig. 4, H-H structure shows the changes in average dispersion on changing inner $(\mathbf{r 1}, \mathbf{a 1})$ and outer parameter $(\mathbf{r}, \mathbf{a}) . \pm 2 \%$ variation in $\mathbf{r 1}$, a1 results in $\pm 2 \%$ and $\pm 4 \%$ changes in average dispersion respectively whereas $\Delta \mathrm{D}$ increases more than $70 \%$ and higher than $100 \%$ respectively. \pm $2 \%$ variation in $\mathbf{r}$, a results in $\pm 5 \%$ and $\pm 8 \%$ changes in average dispersion respectively whereas $\Delta \mathrm{D}$ increases more than $60 \%$ and more than $85 \%$ respectively.

In fig. 5, C-C structure shows the changes in average dispersion on changing inner $(\mathbf{r 1}, \mathbf{d 1})$ and outer parameter $(\mathbf{r}, \mathbf{d}) . \pm 2 \%$ variation in $\mathbf{r 1}$, d1 results in $\pm 3 \%$ and $\pm 2 \%$ changes in average dispersion respectively while $\Delta \mathrm{D}$ increases more than $70 \%$ and more than $60 \%$ respectively. $\pm 2 \%$ 
variation in $\mathbf{r}, \mathbf{d}$ results $\pm 5 \%$ and $\pm 8 \%$ changes in average dispersion respectively whereas $\Delta \mathrm{D}$

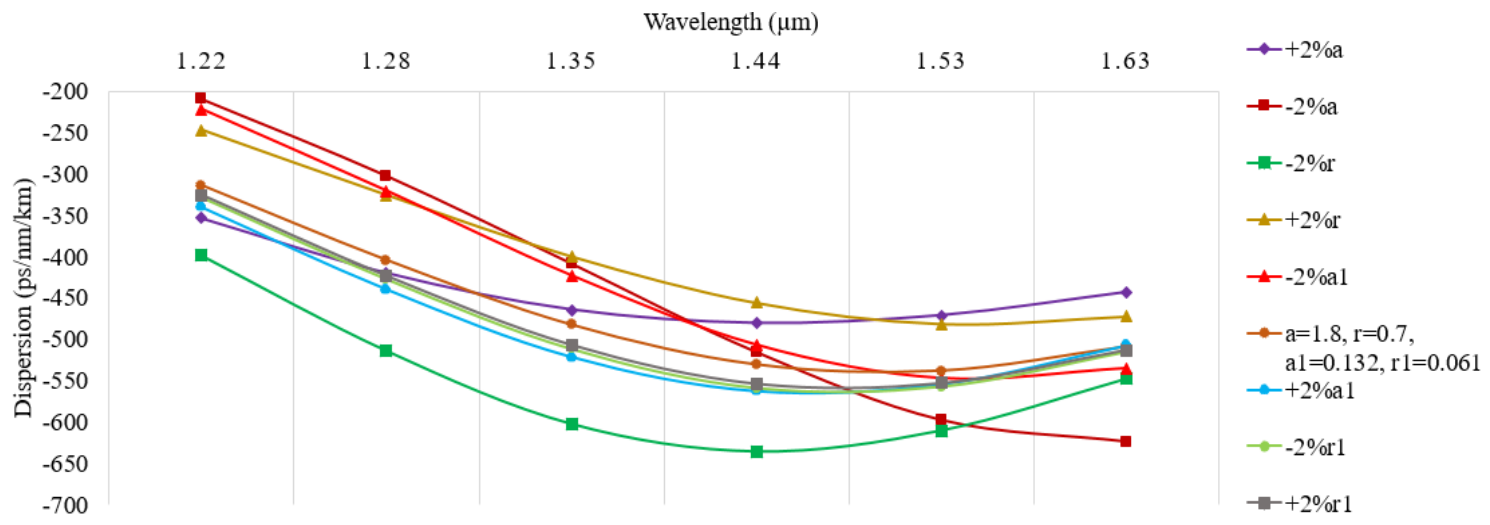

Fig. 4. H-H: Dispersion vs Wavelength

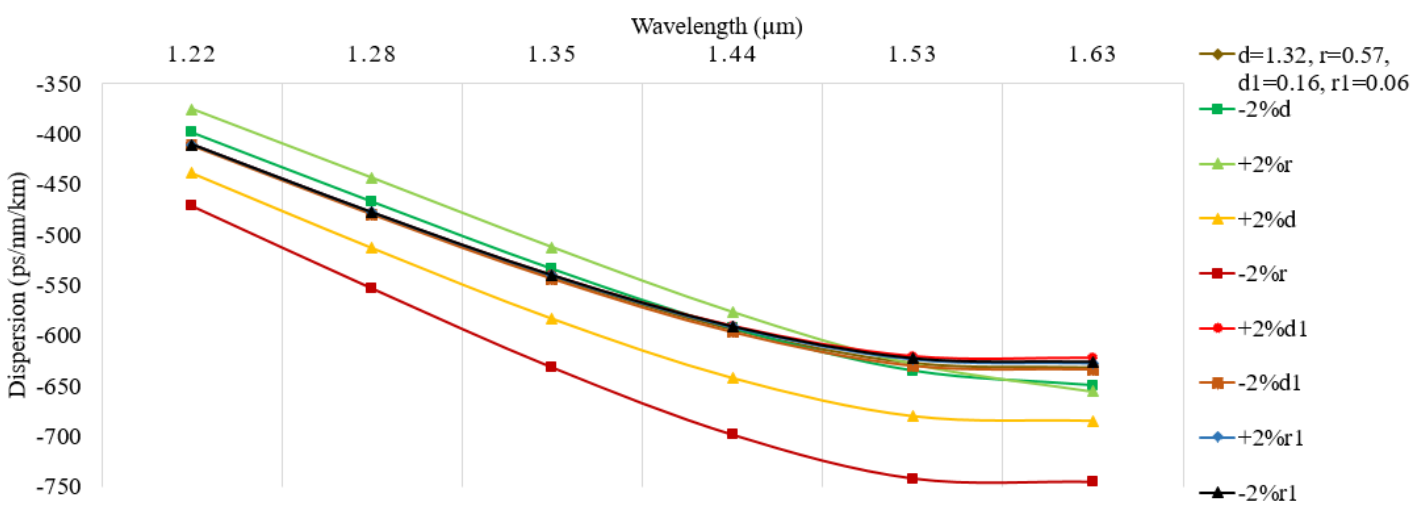

Fig. 5. C-C: Dispersion vs Wavelength

increases more than $60 \%$ and more than $85 \%$ respectively.

In general, while the pitch a or $\mathbf{d}$ of a PCF decreases, the negative dispersion increases whereas increase in $\mathbf{r}$ results in increase in negative dispersion which suggests reduction in effective refractive index.

Effective mode area represents quantitative measurement of the area covered by the fiber's guided mode. Fig. 6, 7, 8 and 9 demonstrate that effective mode area increase with an increase in operating wavelength and corresponding nonlinear coefficient $\gamma$ decreases with an increase in operating wavelength. The calculated nonlinear coefficient of all four proposed structures $\mathrm{C}-\mathrm{H}, \mathrm{H}-\mathrm{H}, \mathrm{H}-\mathrm{C}, \mathrm{C}-\mathrm{C}$ are $11,17.6,18.67,25.08 \mathrm{~W}^{-1} \mathrm{Km}^{-1}$ at $1.55 \mu \mathrm{m}$ respectively with material related nonlinear coefficient is $2.6 \times 10^{-20} \mathrm{~m}^{2} \mathrm{~W}^{-1}$. Four-wave mixing is considered to be harmful for multi channels fiber optic communication system such as WDM, therefore lower nonlinear coefficient from transmission medium is required to reduce four-wave mixing nonlinear effect. Of all the four structures, structure $\mathrm{C}-\mathrm{H}$ exhibit smaller nonlinear coefficient and high effective mode area as compared to remaining three structures. 
Journal of Microwaves, Optoelectronics and Electromagnetic Applications, Vol. 15, No. 4, December 2016372 DOI: http://dx.doi.org/10.1590/2179-10742016v15i4720

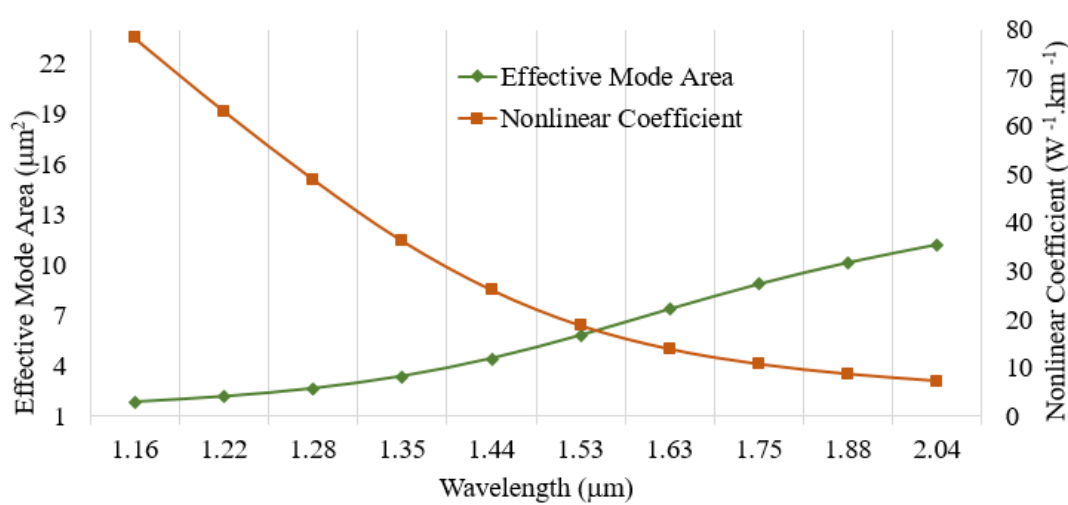

Fig. 6. H-H: Effective Mode Area \& Nonlinear Coefficient

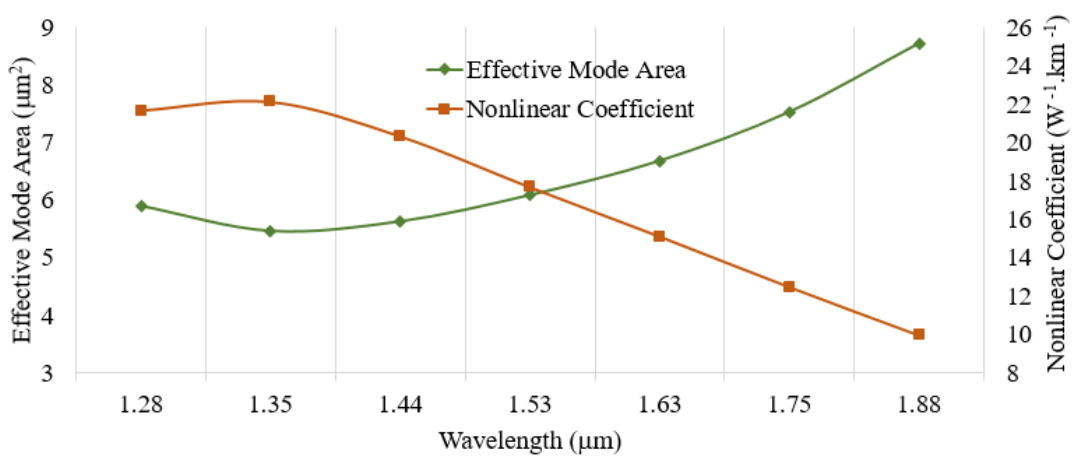

Fig. 7. H-C: Effective Mode Area \& Nonlinear Coefficient

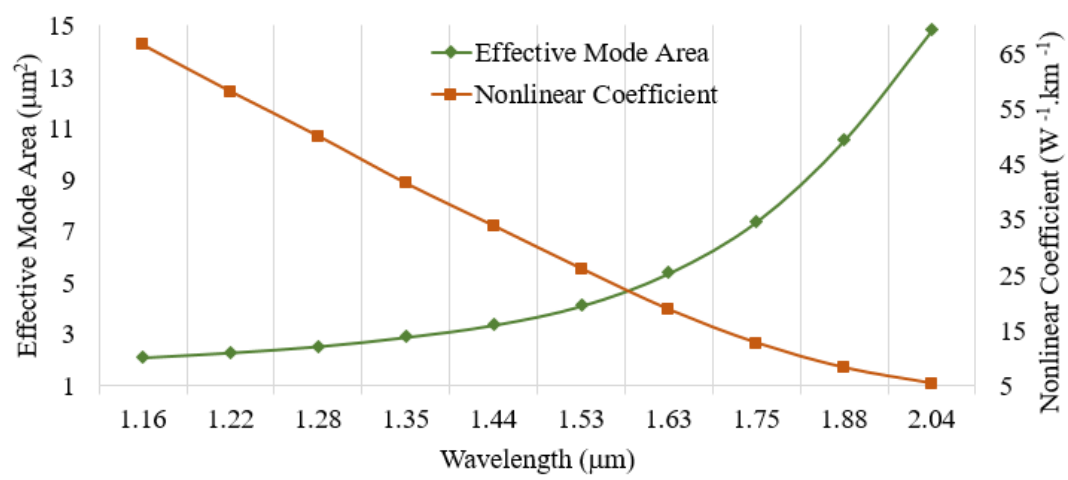

Fig. 8. C-C: Effective Mode Area \& Nonlinear Coefficient 
Fig. 10 shows the comparison of dispersion values for four different structures for the optimized geometric parameters (as shown in table I), against operating wavelength range of 1.16 to $1.83 \mu \mathrm{m}$. It is clearly evident from the figure that structure $\mathrm{C}-\mathrm{H}$ produces larger and flattened negative dispersion as compared to other three structures. The dispersion value of the optimized $\mathrm{C}-\mathrm{H}$ structure is coming around $-704.63 \mathrm{ps} / \mathrm{nm} / \mathrm{km}$ with $\Delta \mathrm{D} \sim 12.5 \mathrm{ps} / \mathrm{nm} / \mathrm{km}$ (range from -689.1 to $-708.8 \mathrm{ps} / \mathrm{nm} / \mathrm{km}$ ) in the wavelength range from 1.22 to $1.57 \mu \mathrm{m}$. $\mathrm{C}-\mathrm{H}$ structure shows better flattened negative dispersion for the wavelength range 1.22 to $1.57 \mu \mathrm{m}$ as compared to other three structures. Beyond $1.57 \mu \mathrm{m}$ till $1.675 \mu \mathrm{m}$, the dispersion value rises to $-560 \mathrm{ps} / \mathrm{nm} / \mathrm{km}$. However, still this result is better as compared

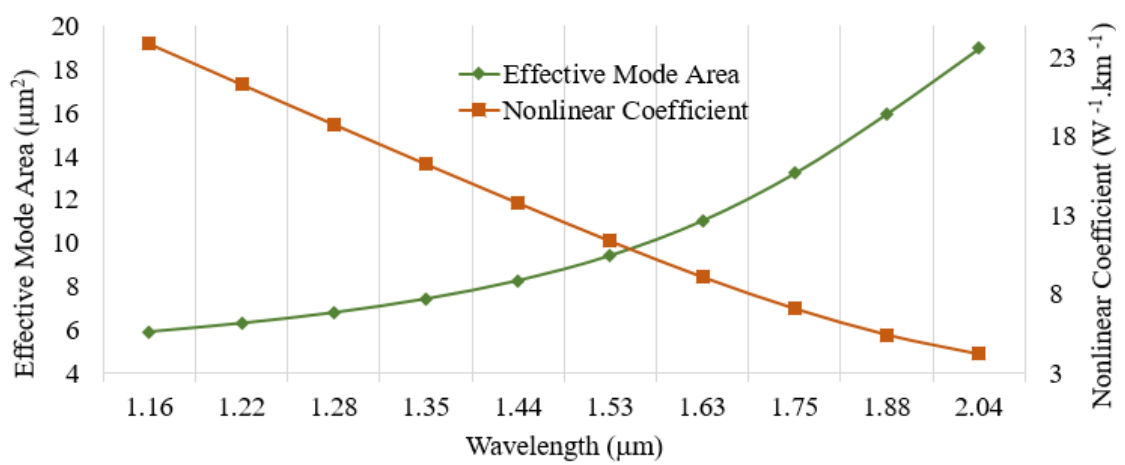

Fig. 9. C-H: Effective Mode Area \& Nonlinear Coefficient

to previous best results. Although the total flattened dispersion is not in the entire communication bands still this high negative dispersion is sufficient to compensate PCF and useful in future application of WDM optical communication system.

To compensate residual dispersion, both large negative dispersion and small dispersion slope value are required. From these different proposed structures, $\mathrm{C}-\mathrm{H}$ structure has dispersion slope range from -0.26 to $0.53 \mathrm{ps} / \mathrm{nm}^{2} / \mathrm{km}$ over the $1.22 \mu \mathrm{m}$ to $1.57 \mu \mathrm{m}$ wavelength at optimum parameters. At $1.29 \mu \mathrm{m}$ to $1.38 \mu \mathrm{m}$ the slope is $0.05 \mathrm{ps} / \mathrm{nm}^{2} / \mathrm{km}$.

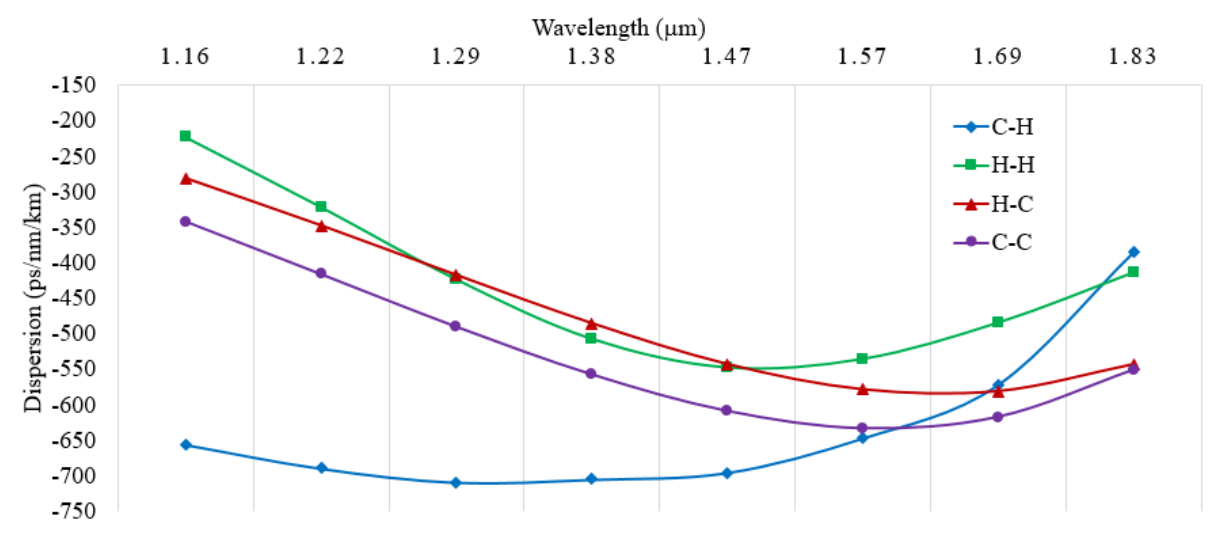

Fig. 10. Structure comparison Dispersion v/s Wavelength 
Fig. 11 shows that there is very low or near zero confinement loss in any of the four proposed different structures. Confinement loss depends on imaginary part of refractive index as given in equation 2. Light confinement is better around core region as r/a ratio increases. As the radius of air hole at cladding region increases it prevent light dissipation into cladding region and hence increases light confinement. All four structures are able to guide light with near zero confinement loss. The confinement loss for $\mathrm{C}-\mathrm{H}$ structure is $2 \times 10^{-8} \mathrm{~dB} / \mathrm{cm}$ at wavelength $1.55 \mu \mathrm{m}$ when $\mathrm{N}=5$. Since the confinement loss is depending on cladding region, this loss can further be reduced by increasing

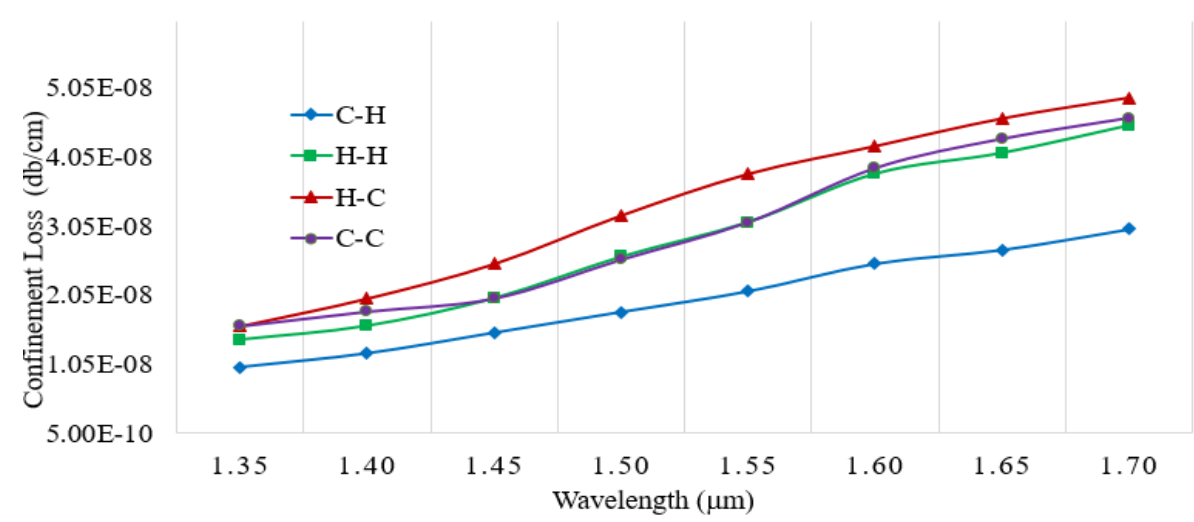

Fig. 11. Confinement Loss comparison for all 4 structures

number of air hole rings and the radius of air holes [12].

Fig. 12 compares the effective mode area against $\mathbf{r 1 / a 1}$ ratio in case of inner hexagon structure and $\mathbf{r 1 / d 1}$ in case of inner circular structure at wavelength $1.55 \mu \mathrm{m}$. Structure $\mathrm{C}-\mathrm{H}$ shows larger increase in effective mode area corresponding to increase in $\mathbf{r} \mathbf{1} / \mathbf{a} 1$ or $\mathbf{r} 1 / \mathbf{d} \mathbf{1}$ ratio as compared to other three

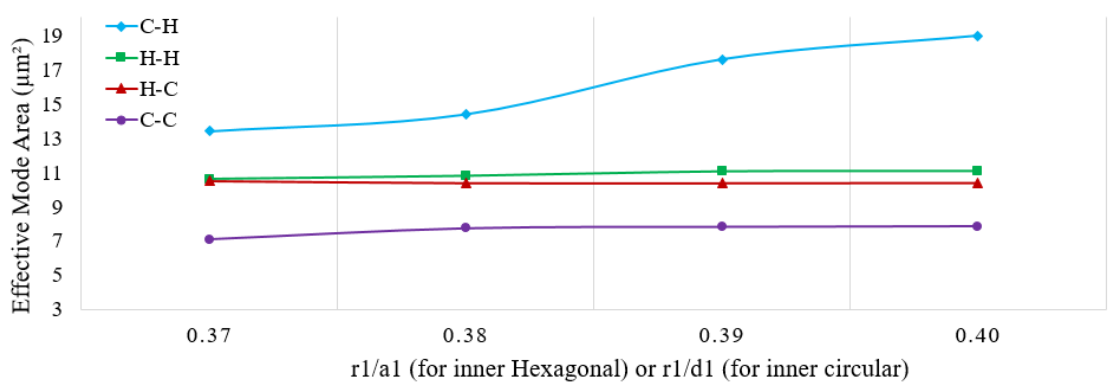

Fig. 12. Comparison of Effective Mode Area

structures.

Fig.13 shows the effect of change in r/a ratio on effective mode area for any given value of $\mathbf{r} 1 / \mathbf{d} \mathbf{1}$ for $\mathrm{C}-\mathrm{H}$ structure. It clearly shows that effective mode area increases with reduction in r/a ratio for

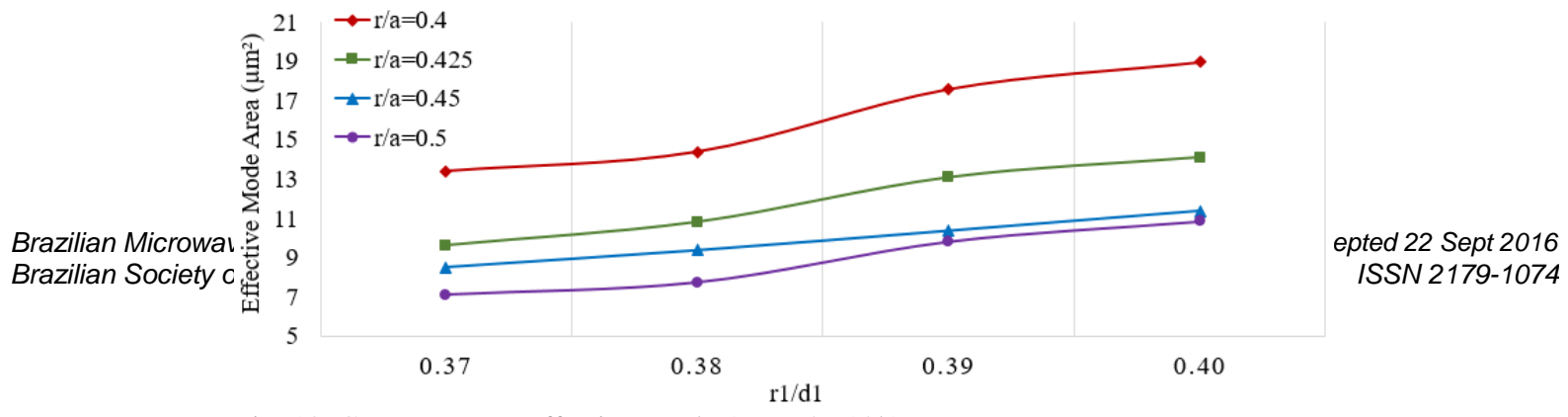

Fig. 13. C-H structure: Effective Mode Area v/s r1/d1 
any given ratio of $\mathbf{r 1 / d 1}$. Also there is increase in effective mode area for any given value of r/a ratio as the ratio $\mathbf{r} 1 / \mathbf{d} 1$ increases. This is due to the fact that the average effective refractive index at core region reduces with respect to increase in $\mathbf{r 1}$. Hence light confinement in core region is weaker which means it is forced out to the cladding region by inner array of circular holes. This also means that light confinement is better around the core region as the ratio r/a increases.

Fig. 14 compares real and imaginary part of effective refractive index against wavelength for all four different structures. Real part of refractive index is shown by solid lines whereas imaginary part of refractive index is shown by dashed lines. Structure C-H shows higher effective refractive index than the other remaining structures. Linear form of the real part of the refractive index shows that the light is confined and non-dispersive in nature. Imaginary part of refractive index is nearly zero which leads to near zero confinement loss based on equation 2 .

The absolute birefringence of all these structures are $\sim 10^{-8}$ at $1.55 \mu \mathrm{m}$ wavelength. This is negligible value due to symmetric structures, hence the effective index of the x-polarized and $y$ polarized guided mode is degenerated [13].

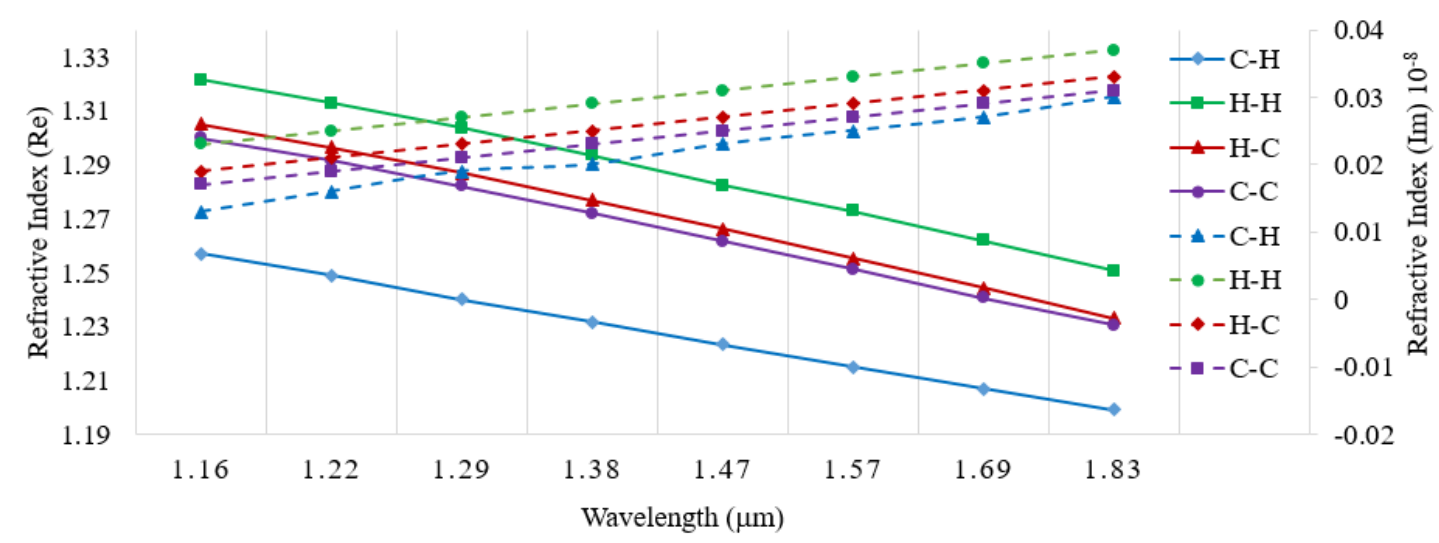

Fig. 14. Refractive Index (Re) v/s Wavelength Comparison

Table II shows comparison of dispersion, effective mode area and nonlinear coefficient obtained earlier by different research scholars.

TABLE II SUMMARY OF THE PREVIOUS RESULTS

\begin{tabular}{lllll}
\hline Reference & $\begin{array}{l}\text { Spectrum } \\
\text { range }(\boldsymbol{\mu m})\end{array}$ & Dispersion $(\mathbf{p s} / \mathbf{n m} / \mathbf{k m})$ & $\begin{array}{l}\text { Effective } \\
\text { Mode Area } \\
\left(\boldsymbol{\mu m}^{\mathbf{2}}\right)\end{array}$ & $\begin{array}{l}\text { Nonlinear } \\
\text { coefficient } \\
\left(\mathbf{W}^{-1} \mathbf{k m}^{-1}\right)\end{array}$ \\
\hline$[4]$ & 1.48 to 1.63 & -98.3 & - & - \\
{$[5]$} & 1.48 to 1.675 & -179 & 6 & - \\
{$[6]$} & 1.35 to 1.7 & -212 & 5 & - \\
{$[7]$} & 1.35 to 1.675 & -227 & 5 & - \\
{$[8]$} & 1.36 to 1.69 & -457.4 & 9 & 11.26 \\
\hline
\end{tabular}


Table III below shows the range of the dispersion, nonlinear coefficient and Effective mode area obtained in this research for each geometric structure for fixed wavelength range $1.22 \mu \mathrm{m}$ to $1.57 \mu \mathrm{m}$. Dispersion range values shown are based on consideration of $\pm 2 \%$ variation in the geometrical parameters. Effective mode area and nonlinear coefficient values are calculated for nominal dimensions (without consideration of $\pm 2 \%$ variation) of geometric parameters. Structure C-H is able to handle wavelength band $\mathrm{O}+\mathrm{E}+\mathrm{S}+\mathrm{C}$ and beyond up to certain extent on $\mathrm{O}$ band side.

Table III list of the different structures and their parameters

\begin{tabular}{llccl}
\hline Structure & $\begin{array}{l}\text { Dispersion }(\mathbf{p s} / \mathbf{n m} / \mathbf{k m}) \\
\text { (for } \mathbf{1 . 1 5 7} \boldsymbol{\mu m} \text { to } \mathbf{1 . 8 3 3} \boldsymbol{\mu m})\end{array}$ & $\begin{array}{l}\text { Effective Mode } \\
\text { Area } \boldsymbol{\mu m}^{2}(\mathbf{a t} \mathbf{1 . 5 5} \boldsymbol{\mu m})\end{array}$ & $\begin{array}{l}\text { NonLinear Coefficient } \\
\mathbf{W}^{-1} \mathbf{k m}^{-1}(\mathbf{a t} \mathbf{1 . 5 5} \boldsymbol{\mu m})\end{array}$ & $\begin{array}{l}\text { Flattened } \\
\text { dispersion band }\end{array}$ \\
\hline C-H & -655.641 to -700.626 & 9.10 & 11.02 & $\begin{array}{l}\text { O+E+S+C } \\
(1.26 \mu \mathrm{m} \text { to } 1.565 \mu \mathrm{m})\end{array}$ \\
\hline H-H & -222.050 to -547.430 & 6.77 & 17.60 & $\begin{array}{l}\mathrm{E}+\mathrm{S}+\mathrm{C} \\
(1.36 \mu \mathrm{m} \text { to } 1.675 \mu \mathrm{m})\end{array}$ \\
\hline H-C & -280.760 to -542.689 & 7.08 & 18.67 & $\begin{array}{l}\mathrm{C}+\mathrm{L}+\mathrm{U} \\
(1.53 \mu \mathrm{m} \text { to } 1.675 \mu \mathrm{m})\end{array}$ \\
\hline C-C & -341.530 to -633.126 & 5.10 & 25.08 & $\begin{array}{l}\mathrm{C}+\mathrm{L}+\mathrm{U} \\
(1.53 \mu \mathrm{m} \text { to } 1.675 \mu \mathrm{m})\end{array}$ \\
\hline
\end{tabular}

Fig. 15 shows the time - averaged poynting vector profile for two degenerate modes at $1.55 \mu \mathrm{m}$ in propagation direction. Modes profiles of $\mathrm{C}-\mathrm{C}$ and $\mathrm{H}-\mathrm{C}$ structures show that the light propagate towards inner structure, whereas H-H structure shows the less light intensity. It can be observed that the modes profile of C-H structure is more confined around outer region of PCF core. This is due to relatively high refractive index at outer region (cladding) as compared to lower refractive index of
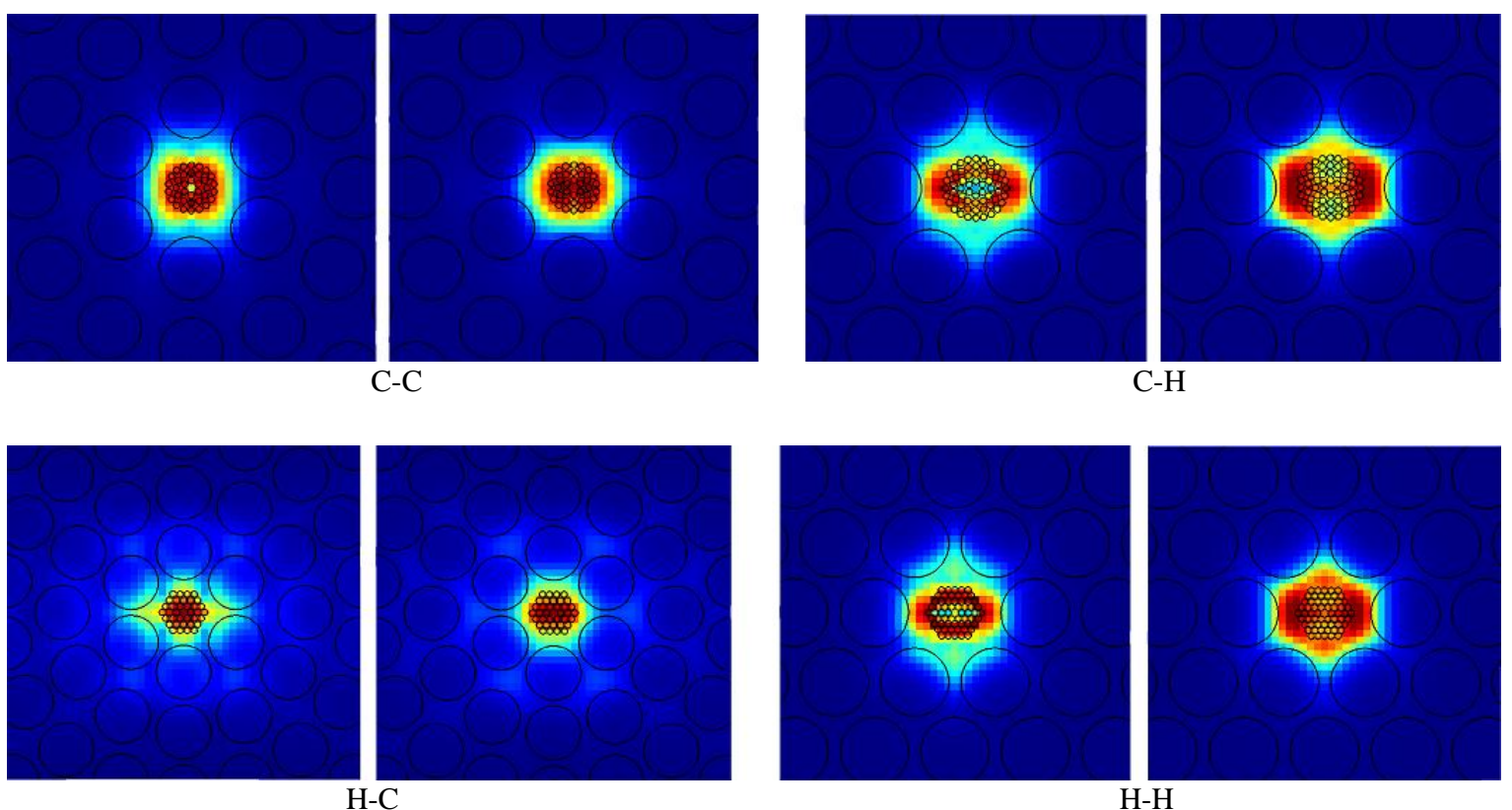

Fig. 15. Time averaged poynting vector profile core region. 
Below given is the comparative study between 2 different structures keeping outer structure shape same against different inner structure. For example, if outer structure is hexagonal then inner structure circular and hexagonal is considered for comparative study. Similarly, comparative study is carried out for 2 different structures keeping inner structure same against different outer structure. This leads to 4 comparative studies which is explained below.

1. Comparison between structures where outer structure is hexagon: $\mathrm{C}-\mathrm{H}$ structure is more suitable for reducing residual dispersion than $\mathrm{H}-\mathrm{H}$ structure. This is because, inner structure is more responsible to reducing residual dispersion. However, C-H structure provides more negative average dispersion than $\mathrm{H}-\mathrm{H}$ structure, as shown in fig. 10.

2. Comparison between structures where outer structure is circular: $\mathrm{C}-\mathrm{C}$ structure gives more linear light propagation than $\mathrm{H}-\mathrm{C}$ structure. Inner circular shape gives more effective area which helps to compensate dispersion as compared to inner hexagonal shape. The confinement loss is also less in C-C as compare to $\mathrm{H}-\mathrm{C}$, as shown in fig. 11.

Above two comparative studies suggest that circular shape at the core region is more preferable over hexagonal shape.

3. Comparison between structures where inner structure is circular: $\mathrm{C}-\mathrm{H}$ structure gives more light confinement than $\mathrm{C}-\mathrm{C}$ structure, the cladding region is responsible to prohibit light to enter into outer region. The effective mode area is more in $\mathrm{C}$ - $\mathrm{H}$ as compared to $\mathrm{C}-\mathrm{C}$ structure, as shown in fig. 12.

4. Comparison between structures where inner structure is hexagonal: $\mathrm{H}-\mathrm{H}$ structure is more preferable over $\mathrm{H}-\mathrm{C}$ structure because of lower confinement loss and better negative dispersion in $\mathrm{H}-\mathrm{H}$ structure as compared to $\mathrm{H}-\mathrm{C}$ structure, as shown in fig. 10.

Above two comparative studies suggest that outer hexagonal structure is more preferable over circular shape.

Based on above four comparative studies we can conclude that $\mathrm{C}-\mathrm{H}$ structure is more preferable over other three structures.

Overall the proposed optimum $\mathrm{C}-\mathrm{H}$ structure show the best result i.e. higher negative dispersion with effective mode area and nonlinear coefficient as well. In addition, this value is nine time higher than the conventional PCF.

The high negative dispersion can be related to the variation of the fundamental guided mode to the Gaussian profile of conventional PCF mode. As a result, proposed optimum structure is more attractive for dispersion compensation at low cost and shorter fiber length as compare to conventional PCF.

While using standard stack and draw fabrication technique [13] for producing air holes in the core region precision control is essential. Fabrication of the proposed structures is possible as these four structures consider $120 \mathrm{~nm}$ and $133 \mathrm{~nm}$ as the diameter of air holes which are larger than the recent trend of fabricating diameter of $110 \mathrm{~nm}$ [14] using standard stack and draw technique. Using 
extrusion technique, it is even possible to fabricate air hole size of the order of $20 \mathrm{~nm}$ [15].

\section{CONCLUSION}

Four different PCF structures with different cross sections such as Circle in Circle, Hexagon in Hexagon, Circle in Hexagon and Hexagon in Circle were investigated for the application of broadband. All structures can be used for different wavelength bands. Structure with cross section of Circle in Hexagon exhibits excellent results as compared to other three structures which can be used for larger wavelength band. C-H structure can be used as a PCF based dispersion compensating fiber over the range of $1.22 \mu \mathrm{m}$ to $1.57 \mu \mathrm{m}$ i.e. $190.95 \mathrm{THz}$ to $245.731 \mathrm{THz}$. It covers $\mathrm{O}$ band to $\mathrm{C}$ band. By varying geometrical parameters of the $\mathrm{C}-\mathrm{H}$ structure, broadband $\mathrm{PCF}$ with large negative dispersion over $350 \mathrm{~nm}$ range and very low confinement loss can be obtained. Besides comparing with previous complicated structures, the design procedure of $\mathrm{C}-\mathrm{H}$ structure is much easier as very few parameters need to be optimized. We believe that the proposed $\mathrm{C}-\mathrm{H}$ structure can be used for future high speed transmission.

\section{ACKNOWLEDGMENT}

The authors wish to thank the anonymous reviewers for their useful comments and constructive suggestions. The authors would like to thank Sardar Patel Institute of Technology Research Centre, Mumbai, India for their guidance in numerical analysis and providing facilities.

\section{REFERENCES}

[1] G.P.Agrawal, Fiber optic communication systems, $2^{\text {nd }}$ ed. Wiley.

[2] Feroza Begum, Yoshinori Namihira ,S.M. Abdur Razzak, Shubi Kaijage, Nguyen HoangHai, Tatsuya Kinjo, Kazuya Miyagi, Nianyu Zou, "Novel broadband dispersion compensating photonic crystal fiber: Application in high-speed transmission systems," Optics and Laser Technology, vol. 41p.p. 679-686,2009.

[3] S. Makoui,M. Savadi-Oskouei,A. Rostami and Z. D. K. Kanani, "Dispersion Flattened Optical Fiber Design for Large Bandwidth and High-speed Optical Communications using Optimization Technique" PIER B, Vol. 13, 21-40, 2009

[4] S. K. Varshney, N. J. Florous, K. Saitoh, M. Koshiba, and T. Fujisawa, "Numerical investigation and optimization of a photonic crystal fiber for simultaneous dispersion compensation over $\mathrm{S}+\mathrm{C}+\mathrm{L}$ wavelength bands," Opt. Commun., vol. 274, pp. 74-79, 2007

[5] M. A. R. Franco, V. A. Serra o, and F. Sircilli, "Microstructured optical fiber for residual dispersion compensation over S+C+L+U wavelength bands", IEEE Photon. Technol. Lett., vol. 20, no. 9, pp. 751-753, May 2008

[6] José Patrocínio da Silva, Diego S. Bezerra, Vitaly F. Rodriguez-Esquerre, Iguatemi Eduardo da Fonseca, and Hugo E. Hernández-Figueroa, Senior Member, IEEE "Ge-Doped Defect-Core Microstructure d Fiber Design by Genetic Algorithm for Residual Dispersion Compensation” IEEE Photon. Technol. Lett., vol. 22, no. 18, Sept 15, 20101337

[7] Md. Asiful Islam and M. Shah Alam, Senior Member, IEEE, "Design of a Polarization-Maintaining Equiangular Spiral Photonic Crystal Fiber for Residual Dispersion Compensation Over E+S+C+L+U Wavelength Bands" IEEE Photon. Technol. Lett., VOL. 24, NO. 11, JUNE 1, 2012

[8] D. C. Tee, M. H. Abu Bakar, N. Tamchek, and F. R. Mahamd Adikan "Photonic Crystal Fiber in Photonic Crystal Fiber for Residual Dispersion Compensation Over E + S+ C+ L+ U Wavelength Bands" IEEE Photon. Technol. Lett., VOL. 5, NO.3, JUNE 1, 2013.

[9] M.H.Waghmare, Dr. K.T.V.Reddy "Analysis of Photonic Bandgap Structure in 2-D hexagonal and square lattice structure of Photonic Crystal” IEEE Explore Digital Library Proc. ICACACT-2014

[10] R.P. Photonics buyer's guide https://www.rp-photonics.com/buyersguide.html

[11] F. Poletti, V. Finazzi, T. M. Monro, N. Broderick, V. Tse, and D. J. Richardson, "Inverse design and fabrication tolerances of ultra-flattened dispersion holey fibers", Opt. Exp., vol. 13, no. 10, pp. 3728-3736, May 2005.

K. Saitoh, M. Koshiba, T. Hasegawa, and E. Sasaoka, B "Chromatic dispersion control in photonic crystal fibers: Application to ultra-flattened dispersion”, Opt. Exp., vol. 11, no. 8, pp. 843-852, Apr. 2003.

[12] Y. Ruan, S. Afshar, and T. M. Monro, "Light enhancement within nanoholes in high index contrast nanowires", IEEE Photon. J., vol. 3, no. 1, pp. 130-139, Feb. 2011

[13] J. C. Knight, T. A. Birks, P. St. J. Russell, and D. M. Atkin, "All-silica single-mode optical fiber with photonic crystal cladding”, Opt. Lett., vol. 21, no. 19, pp. 1547-1549, Oct. 1996. 
Journal of Microwaves, Optoelectronics and Electromagnetic Applications, Vol. 15, No. 4, December 2016379 DOI: http://dx.doi.org/10.1590/2179-10742016v15i4720

[14] G.S. Wiederhecker, C.M. B. Cordeiro, F.Couny,F.Benabid, S. A. Maier, J. C.Knight, C.H.B. Cruz, andH.L. Fragnito, B "Field enhancement within an optical fibre with a subwavelength air core",Nat.Photon.,vol.1,no.2,pp.115-118,2007.

[15] Y. Ruan, H. Ebendorff-Heidepriem, V. Afshar, and T. M. Monro, "Light confinement within nanoholes in nanostructured optical fibers", Opt. Exp., vol. 18, no. 25, pp. 26 018-26 026, Dec. 2010. 